\title{
Comparative evaluation of three image analysis methods for angular displacement measurement in a MEMS microgripper prototype: a preliminary study
}

\author{
Federica Vurchio ${ }^{1}$, Giorgia Fiori ${ }^{1}$, Andrea Scorza ${ }^{1}$, Salvatore A. Sciuto ${ }^{1}$ \\ ${ }^{1}$ Engineering Department, Roma Tre University, Rome, Italy
}

\begin{abstract}
The functional characterization of MEMS devices is relevant today since it aims at verifying the behavior of these devices, as well as improving their design. In this regard, this study focused on the functional characterization of a MEMS microgripper prototype suitable in biomedical applications: the measurement of the angular displacement of the microgripper comb-drive is carried out by means of two novel automatic procedures, based on an image analysis method, SURF-based (Angular Displacement Measurement based on Speeded Up Robust Features, ADM SURF $_{\text {) }}$ and FFT-based (Angular Displacement Measurement based on Fast Fourier Transform, ADM FFT $_{\text {) }}$ method, respectively. Moreover, the measurement results are compared with a Semi-Automatic Method (SAM), to evaluate which of them is the most suitable for the functional characterization of the device. The curve fitting of the outcomes from SAM and ADM surF, showed a quadratic trend in agreement with the analytical model. Moreover, the ADMSURF measurements below $1^{\circ}$ are affected by an uncertainty of about $0.08^{\circ}$ for voltages less than $14 \mathrm{~V}$, confirming its suitability for microgripper characterization. It was also evaluated that the ADM $\mathrm{FFT}$ is more suitable for measurement of rotations greater than $1^{\circ}$ (up to $30^{\circ}$ ), with a measurement uncertainty of $0.02^{\circ}$, at $95 \%$ of confidence level.
\end{abstract}

Section: RESEARCH PAPER

Keywords: Microgripper; MEMS; microactuators; displacement measurements; characterization

Citation: Federica Vurchio, Giorgia Fiori, Andrea Scorza, Salvatore Andrea Sciuto, Comparative evaluation of three image analysis methods for angular displacement measurement in a MEMS microgripper prototype: a preliminary study, Acta IMEKO, vol. 10, no. 2, article 17, June 2021, identifier: IMEKOACTA-10 (2021)-02-17

Section Editor: Ciro Spataro, University of Palermo, Italy

Received January 18, 2021; In final form April 22, 2021; Published June 2021

Copyright: This is an open-access article distributed under the terms of the Creative Commons Attribution 3.0 License, which permits unrestricted use, distribution, and reproduction in any medium, provided the original author and source are credited.

Corresponding author: Federica Vurchio, e-mail: federica.vurchio@uniroma3.it

\section{INTRODUCTION}

MEMS devices (Micro-electro-mechanical systems) represent a category of sensors and actuators widely used in the most varied fields of technology, from automotive to micro assembly for photonics and RF application, microphones, microfluidic device, gyroscopes, chemical sensors for microfluidics systems, lab-on-chip systems and complex actuation systems [1]. One of the most promising fields of application is undoubtedly the biomedical one, such as biology [2],[3] and microsurgery [4]-[6]. Microgrippers are a particular class of MEMS devices, able to handle objects, including cells and molecules that have micrometric dimensions. Nowadays, there are few works concerning the characterization of devices such as microgrippers, even if the study of the metrological and performance characteristics would be of great help for the optimization of the prototypes and the improvement of their performances. In this study, a set of images have been acquired by means of a trinocular optical microscope and processed by means of three different methods implemented ad hoc in Matlab environment: the Semi-Automatic (SAM), the SURF-based (Angular Displacement Measurement based on Speeded Up Robust Features, ADM $_{\text {SURF }}$ ) and the FFT-based (Angular Displacement Measurement based on Fast Fourier Transform, ADM ${ }_{\mathrm{FFT}}$ ). A comparison among the abovementioned methods has been made to estimate the angular displacement of a MEMS microgripper prototype comb-drive for biomedical applications. SemiAutomatic Method (SAM) already widely described by the authors in [7]-[9], is based on template-matching, and it is able to evaluate the rotation and both the gripper and the angular displacement of a microgripper. Its main limitations are the high computational costs and the operator dependence. 
The above issues have been deepened in this work, starting from the previous study presented in [10]. In Section 2, the materials and methods are described, with particular reference to the experimental setup and the measurement protocol used for the digital image acquisition. Due to the limitation encountered in SAM previously proposed [7]-[9], in Subsection 2.1, the authors propose a new version of the SAM, in which novel tests have been implemented to quantify the uncertainty contribution introduced by the operator in the angular displacement measurement of a microgripper comb-drive prototype; in Subsections 2.2 and 2.3 the authors described two novel and automatic methods and their application for the measurement of the comb-drive angular displacement: the $\mathrm{ADM}_{\mathrm{SURF}}$, based on the SURF algorithm [11], and the $\mathrm{ADM}_{\mathrm{FFT}}$, that is an application of 2D Fast Fourier Transform (FFT) to digital images [12]-[16]. In Section 3, the procedure for estimating the sources of uncertainty of the three measurement methods is described and a comparison and the evaluation of the outcomes obtained through the three abovementioned methods have been carried out and discussed to identify which of the three implemented methods is the best suitable for the characterization of the MEMS device. Finally, in Section 4 and 5, the results of our study are illustrated, and the conclusions presented.

\section{MATERIALS AND METHODS}

In this section the main components of the experimental setup have been described together with a detailed overview of the three implemented methods; in particular, the SURF-based and the FFT-method have been proposed as alternative methods to the Semi-Automatic one for the measurement of the angular displacement of the comb-drive.

The device under examination is a microgripper prototype (Figure 1), which is part of a project concerning the metrological and performance characterization of a new class of MEMS devices for biomedical applications [17]-[21]. These devices mainly consist of capacitive electrostatic actuators (i.e., the comb-drives shown in Figure 2) and particular hinges called Conjugate Surfaces Flexural Hinges (CSFH) [22], which allow the mechanical movement of the tips located on the end of the device. The images have been acquired through a NB50TS trinocular light microscope equipped with a 6MP camera. The device has been positioned on an instrumented stage with micrometric screws and powered through a HP E3631A power supply. The latter is electrically connected to the device by means of a coaxial cable and tungsten needles put in contact with the electrical connections of the device. The voltage has been brought to the electrical connections by means of two micropositioners that allow the tungsten needle movement along the

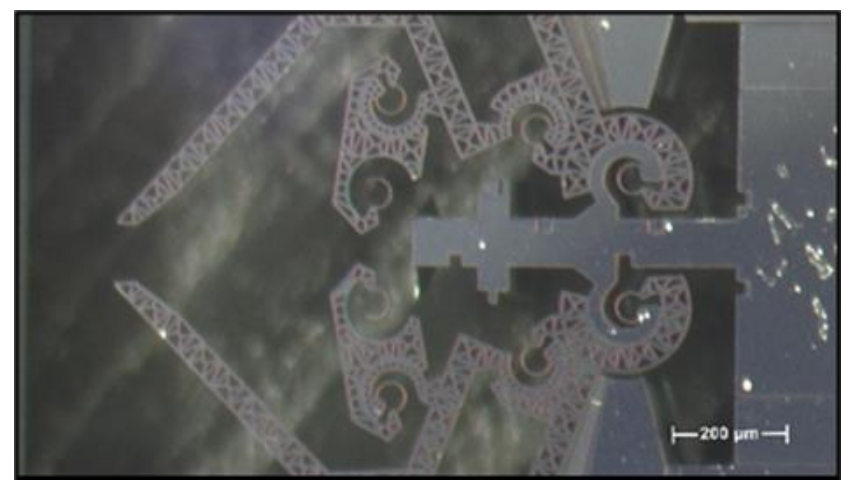

Figure 1. Microgripper prototype.

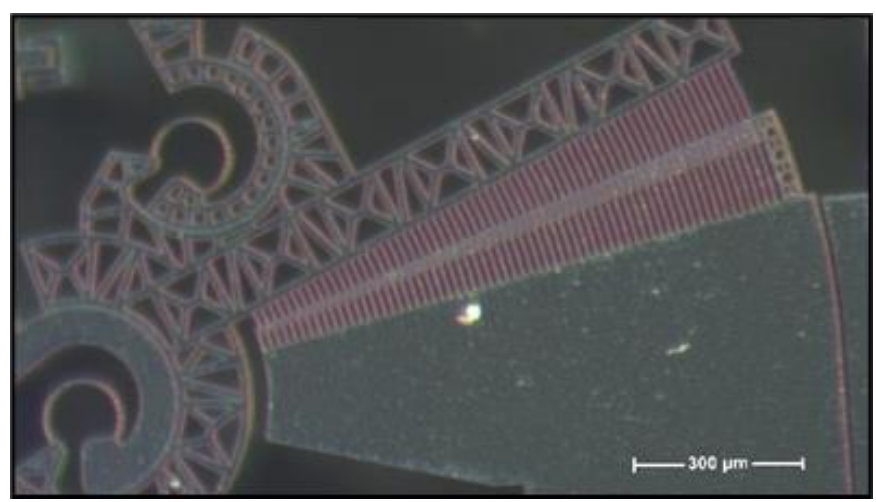

Figure 2. The comb-drive.

three axes, $x, y$, and $z$. A set of 30 images has been collected for each applied voltage with a $2 \mathrm{~V}$ step (i.e., $0 \mathrm{~V}, 2 \mathrm{~V}, 4 \mathrm{~V}, \ldots 24 \mathrm{~V}$ ).

\subsection{Semi-Automatic based method (SAM)}

The first method used in this study, has been the SemiAutomatic one, which for clarity we will call $S A M_{a}$, widely described in [7],[8] and used in [9]. As illustrated in [7]-[9], the method introduces a measurement uncertainty contribution which corresponds to $0.02^{\circ}$, at $95 \%$ confidence level, evaluated by means of Monte Carlo simulation. Moreover, the software requires high computational costs and the uncertainty analysis of the preliminary results obtained with the $S A M_{a}$ was previously carried out partially [7]-[10], assuming the uncertainty component introduced by the operator's subjectivity; for this reason, in this study further tests were carried out to better evaluate the above contribution. The test on the $S A M_{a}$, in fact, consists, in its first part, of a selection by the operator of four points and of a Region of Interest (ROI) on the image; to evaluate the dispersion in the selection of these points, in the new version of Semi-Automatic method, called $S A M_{b}$, ten different observers were asked to identify both the four points and the $\mathrm{ROI}$ in an image of the comb-drive, for a number of times equal to 30. In particular, for the four points, the $\mathrm{x}$ and $\mathrm{y}$ coordinates on the image were considered and for the ROI, the coordinates of the top left vertex ( $x$ and $y$ ), its length and width (each of them expressed in pixels), as can be seen in Figure 3.

\subsection{Speeded Up Robust Features based method (SURF)}

An automatic method based on Speeded Up Robust Features (SURF) has been implemented to measure the angular displacement of the comb-drive ( $\left.\mathrm{ADM}_{\mathrm{SURF}}\right)$, as already described in [23]. It is an interest point detector and descriptor, used in

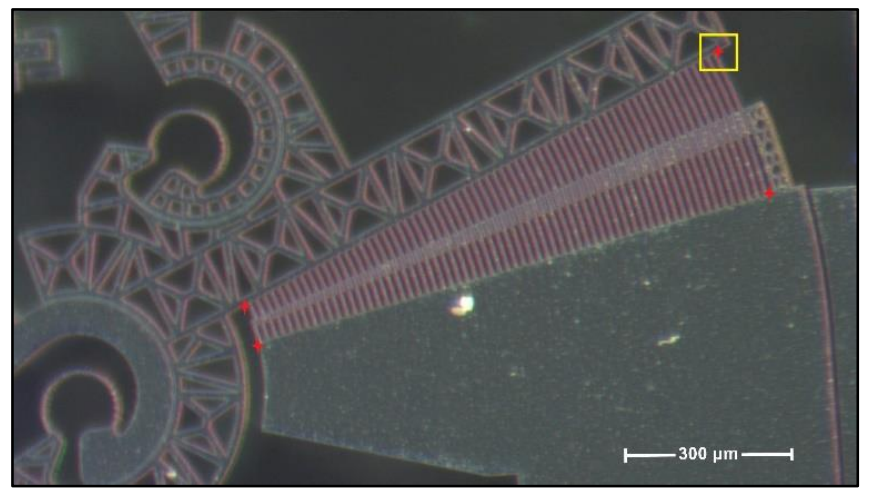

Figure 3. Four points (red cross) and ROI (yellow square) selection on the comb-drive image. 
many applications including image registration, object recognition and 3D reconstruction [24]. The main advantage of this method is mainly the computational cost reduction; in fact, as illustrated in [11], a significant reduction in image processing time has been observed thanks to the complexity reduction of the descriptor, without altering the performance in terms of repeatability, noise robustness, detection errors, geometric and photometric deformation. The in-house method consists of three main steps:

1) finding interest points on the image; in particular, a $\mathrm{ROI}_{0 \mathrm{~V}}$ is selected on the first image Img $\mathrm{I}_{0}$, that corresponds to $0 \mathrm{~V}$ power supply, and it is important that this selected area is chosen by the operator in an image area where the movement of the combdrive is visible; the coordinates of the selected ROI are saved and used to select the ROIs (i.e. $\mathrm{ROI}_{2 \mathrm{~V}}, \mathrm{ROI}_{4 \mathrm{~V}}, \mathrm{ROI}_{6 \mathrm{~V}}, \ldots, \mathrm{ROI}_{24 \mathrm{~V}}$ ) of all the subsequent images $\operatorname{Img}_{2 \mathrm{~V}}, \operatorname{Img}_{4 \mathrm{~V}}, \operatorname{Img}_{6} \mathrm{~V}, \ldots, \operatorname{Img}_{24 \mathrm{~V}}$ After that, the algorithm finds the interest points on each selected ROI.

2) building a descriptor for the representation of the interest points; for example, in this case they are the red circles for the first image and the green crosses for all the others (Figure 4).

3) matching the various descriptors found on the images; by using a geometric transform, the object position on the images can be obtained and therefore it has been possible go back to its relative rotation referred to each applied voltage.

\subsection{Fast Fourier Transform based method (FFT)}

This method is based on the application of the Fourier transform to digital images. As shown in Figure 5, the combdrives of the microgripper have a particular periodic pattern; also, if an image consists of an array of uniformly spaced parallel straight lines, the Fourier transform of the combination will be the convolution of their respective Fourier transform. The result will be a string of impulses (see Figure 6), with a separation equal to the reciprocal of the spacing of the lines and in a direction perpendicular to these parallel lines [12],[13]. This last feature has been used to estimate the angular aperture of the comb-drive: in fact, for each angular opening, the corresponding pattern of the comb-drive will take a different direction and consequently, also the position of the impulses will change and assume a different direction each time. Therefore, for each angular opening, and for each image, there will be a series of points in different directions; subsequently a least squares approximation has been used to find the linear polynomial function that best approximates these points from which the angular coefficient of the straight line is obtained and therefore the opening angle of the comb-drive.
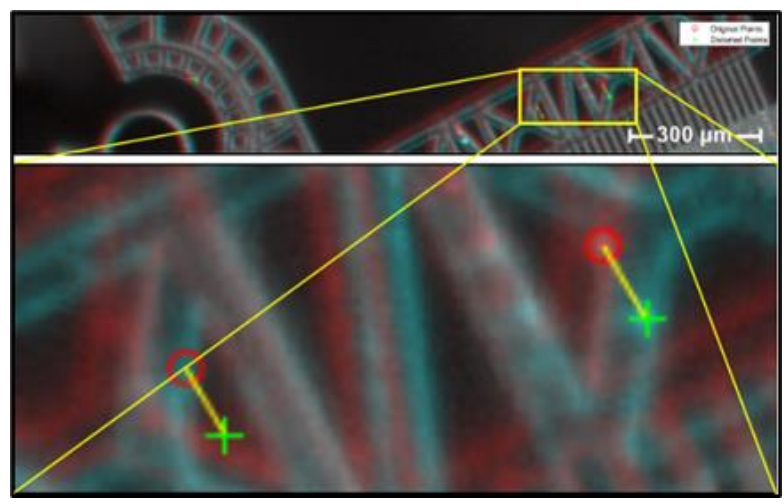

Figure 4. Interest points descriptor of the first image (red circles) and of other images (green cross).
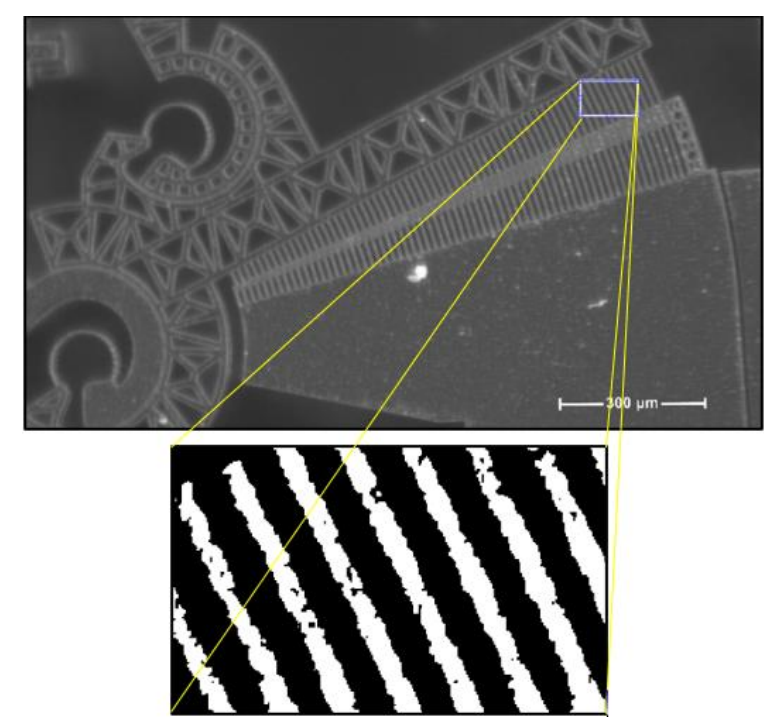

Figure 5. Comb-drive pattern.

As previously noted in [10], the major limitation associated with this procedure is due to the inability of the $\mathrm{ADM}_{\mathrm{FFT}}$ to measure angular displacements less than a tenth of a degree, typical of MEMS devices for biomedical applications such as microgrippers. However, some microgrippers actuated by rotary comb-drive, as those studied in this work, are powered with voltages much higher than $30 \mathrm{~V}$ [25],[26] and therefore it was considered relevant to define whether this method could be used for the characterization of other MEMS devices. In order to evaluate the limit of applicability of the $\mathrm{ADM}_{\mathrm{FFT}}$, we proceeded in this way: once an image that presented a pattern like the one shown in Figure 5 was identified, it was rotated of a quantity reported in Table 1, where $\mathrm{SET}_{1}$ and $\mathrm{SET}_{2}$ correspond to two set of rotation, the first consisting of rotations less than one degree, the second higher than one degree; in particular, the rotation values of the first set correspond to the measurements obtained from the images acquired during the experimental campaign, using the SAM.

\section{UNCERTAINTY ANALYSIS}

In order to make a comparison among the three different image analysis methods, it is necessary to estimate the main uncertainty sources introduced by the measurement systems. It is important to underline that the experimental setup is the same, except for the three different methods. Following the procedure adopted in [7], Type A and Type B uncertainties will be combined [27], as follows (1):

$$
\delta_{T}=\sqrt{\delta_{A}^{2}+\delta_{B}^{2}}
$$

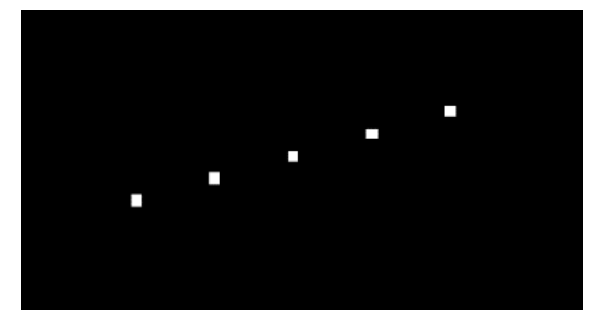

Figure 6. Example of Fourier transform applied to images properly filtered, constituted by a string of impulses. 
Table 1. Rotation values.

\begin{tabular}{lcccccccccccc}
\hline $\mathrm{SET}_{1}$ in $^{\circ}$ & 0.007 & 0.032 & 0.070 & 0.120 & 0.194 & 0.277 & 0.379 & 0.497 & 0.631 & 0.777 & 0.939 & 1.118 \\
\hline $\mathrm{SET}_{2}$ in $^{\circ}$ & 1 & 2 & 3 & 4 & 5 & 7 & 10 & 13 & 16 & 20 & 25 & 30 \\
\hline
\end{tabular}

where Type A uncertainty, $\delta_{A}$, has been calculated directly from the standard deviation of the experimental data, while Type B uncertainty, $\delta_{B}$, has been obtained considering the uncertainties due to the power supply (evaluated from the datasheet), the optical system [7]-[9], the angle measurement, which uncertainty contribution has been assessed by means of a Monte Carlo simulation [28]-[29] in order to estimate the uncertainty related to the three implemented method.

Considering the SAM, in order to simulate the uncertainty of the operator's point selection and therefore evaluate the algorithm uncertainty, a Monte Carlo simulation with $10^{4}$ iterations has been performed. In Table 2 , the variables $x, y$ and ROI with their assigned distributions and their standard deviations have been reported, in order to estimate the uncertainty introduced by the method. This contribution has been evaluated for each angular displacement of the comb-drive (i.e. $\delta_{\alpha_{0-2 \mathrm{~V}}}, \delta_{\alpha_{0-4 \mathrm{~V}}}, \delta_{\alpha_{0-6 \mathrm{~V}}}, \ldots, \delta_{\alpha_{0-24 \mathrm{~V}}}$ ), and combined with the Type A uncertainty, following the equation (1). On the other hand, to evaluate the uncertainty introduced by the FFT based method in the measurement of the angular displacement of the comb-drive, the image in Figure 5 has been subjected to different rotations. The contribution of the systematic uncertainty, considered in this procedure, has been evaluated by building a particular 4K (3840 px $\times 2160 \mathrm{px})$ image (Figure 7), rotated by the same quantities reported in Table 1 . This contribution is mainly due to the uncertainty with which the software implements the rotation of the image and therefore into the error that it makes in measuring the angle $\alpha$. Considering that at angles up to $15^{\circ}$ the sine is only about $1 \%$ different and the tangent about $2 \%$ different from the measurement of the angle in radiant [30], the following approximation can be used (2):

$$
\tan \alpha \cong \alpha=\frac{a}{b}
$$

where $a$ and $b$ are the measurements of the segment reported in Figure 7; therefore, for angles less than $15^{\circ}$, the angle measurement relative uncertainty $\delta_{\alpha}$ has been evaluated by the following equation (3):

$$
\frac{\delta_{\alpha}}{\alpha}=\sqrt{\left(\frac{\delta_{a}}{a}\right)^{2}+\left(\frac{\delta_{b}}{b}\right)^{2}}
$$

Table 2. Variables settings in MCS to estimate the uncertainty introduced by the operator's subjectivity.

\begin{tabular}{ccc}
\hline Parameter & Distribution & Standard Deviation in px \\
\hline P1 Coordinate $x$ & & 8 \\
P2 Coordinate $x$ & Gaussian & 10 \\
P3 Coordinate $x$ & 8 \\
P4 Coordinate $x$ & & 9 \\
\hline P1 Coordinate $y$ & & 6 \\
P2 Coordinate $y$ & Gaussian & 7 \\
P3 Coordinate $y$ & & 6 \\
P4 Coordinate $y$ & & 7 \\
\hline ROI Coordinate $x$ & & 15 \\
ROI Coordinate $y$ & Gaussian & 14 \\
ROI width & & 20 \\
ROI height & & 18 \\
\hline
\end{tabular}

where $\delta_{a}$ and $\delta_{b}$ are the measurement uncertainty of segments $a$ and $b$, respectively, which are considered $\pm 1 \mathrm{px}$, and on the other hand, if $\alpha$ is greater than $15^{\circ}$, it can be determined as follows (4):

$$
\alpha=\operatorname{arctg}\left(\frac{a}{b}\right)
$$

and its uncertainty $\delta_{\alpha}$ can be evaluated by equation (5), as

$$
\delta_{\alpha}=\frac{d[\operatorname{arctg}(c)]}{d c} \cdot \delta_{c}
$$

where $c$ is the ratio between the segments $a$ and $b$, while $\delta_{c}$, is the corresponding uncertainty. Once this uncertainty contribution has been evaluated, for each angular displacement, it is then combined following the equation (1).

Once uncertainties have been evaluated, a comparison among the three different set of results will be made, following the procedure adopted in [8] and reported in [31]. In practice, the different methods are able to measure the angular displacement of the comb-drive without significant differences if the following condition is verified (6):

$$
\left|\bar{M}_{1}-\bar{M}_{2}\right| \leq\left(\delta_{T_{1}}+\delta_{T_{2}}\right),
$$

where $\bar{M}_{1}$ and $\bar{M}_{2}$ are the mean values of the measurement results, while $\delta_{T_{1}}$ and $\delta_{T_{2}}$ are the total uncertainty estimate. In particular, if the difference $\left|\bar{M}_{1}-\bar{M}_{2}\right|$ has the same order of magnitude, or even less than, the sum $\left(\delta_{T_{1}}+\delta_{T_{2}}\right)$, then measurements can be considered consistent, within the interval of the experimental uncertainties.

\section{RESULTS AND DISCUSSION}

In this section the outcomes from SAM, ADM $\mathrm{ADM}_{\mathrm{FFT}}$ are reported and commented. The graphs in Figure 8 and in Figure 9, show the results related to the comb-drive angular displacement, expressed as mean value, corresponding to SAM, ADM SURF $_{\text {and }} \mathrm{ADM}_{\mathrm{FFT}}$ respectively.

Table 4 shows the measurement results expressed as the mean

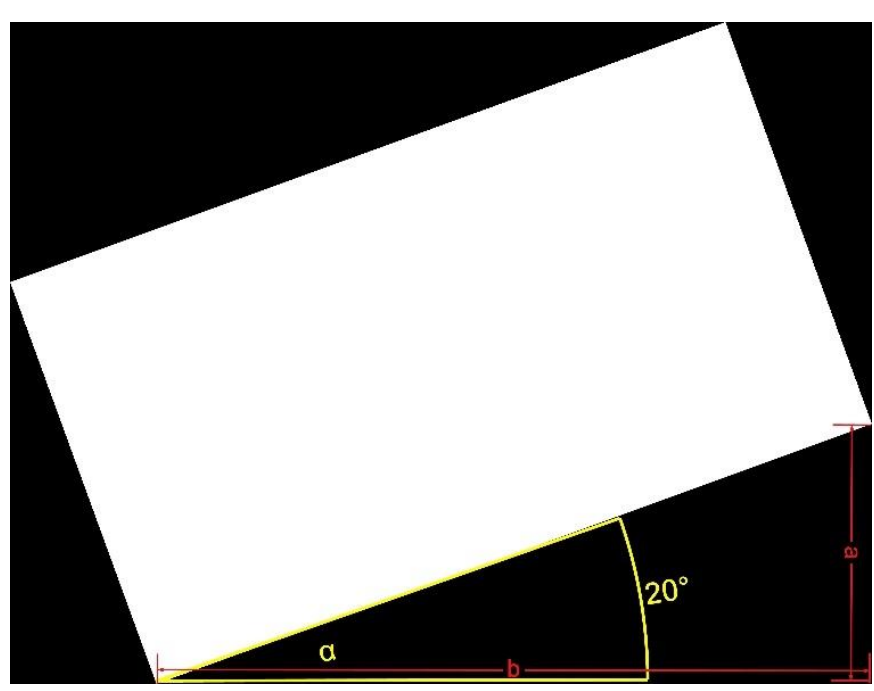

Figure 7. $4 \mathrm{~K}$ image, rotated of $20^{\circ}$ for the estimation of angular rotation uncertainty in FFT-based method. 


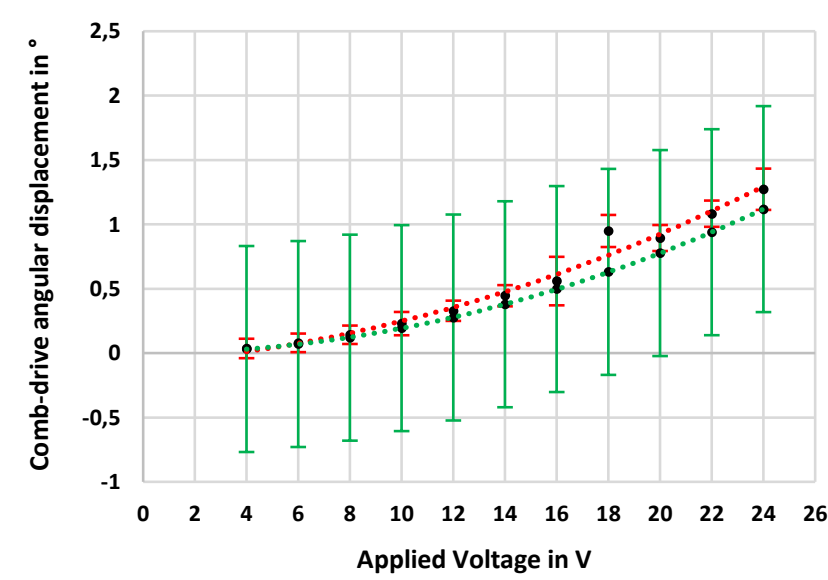

Figure 8. Relationship between angular displacement and applied voltage considering SAM (green dot line) and SURF method (red dot line).

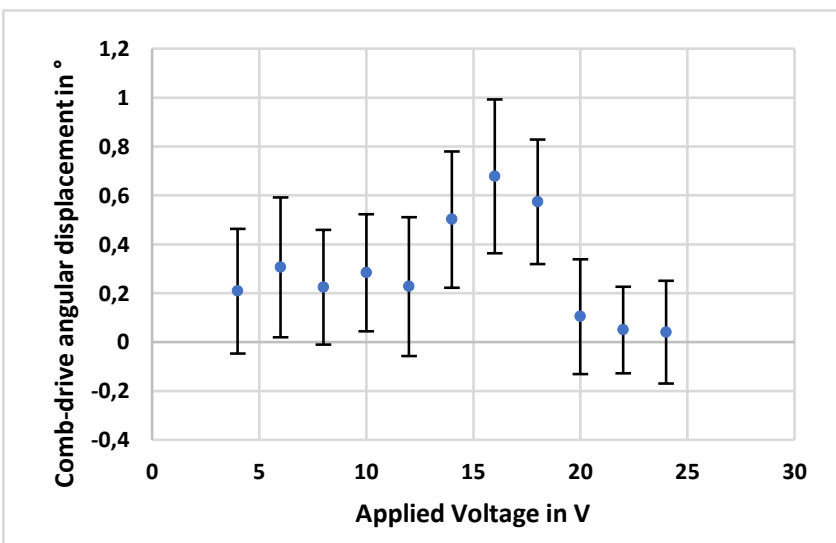

Figure 9. Relationship between angular displacement and applied voltage considering FFT-based method.
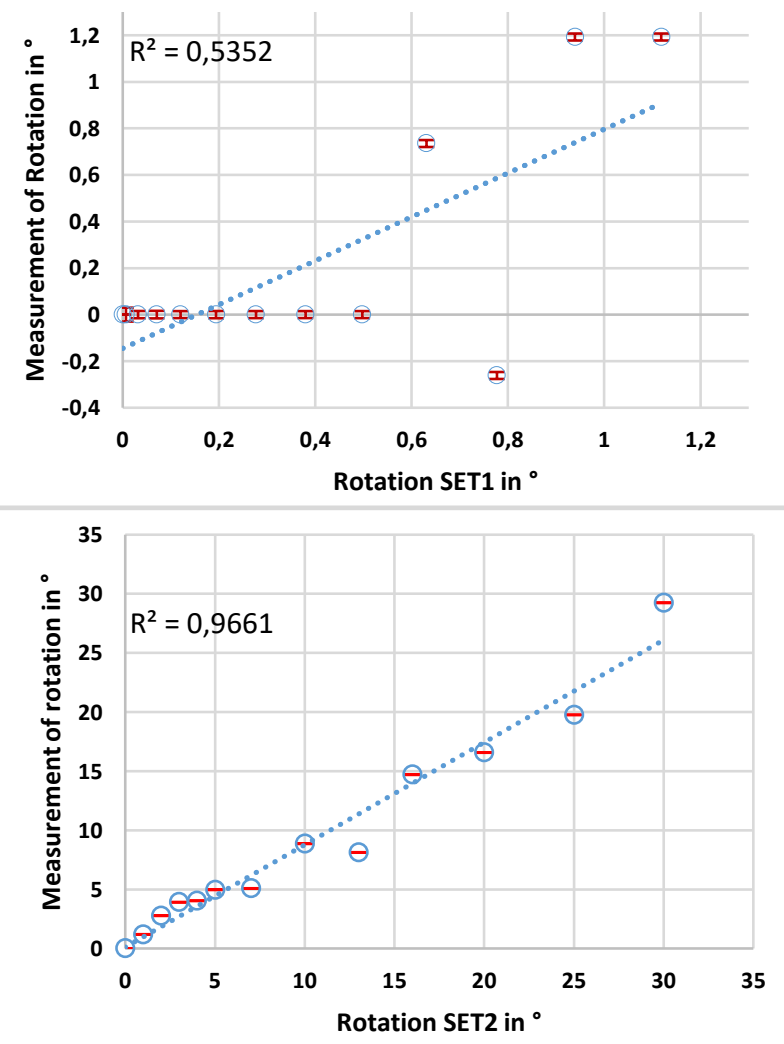

Figure 10. Measurement of rotations less than $1^{\circ}$ (above), and measurement of rotations between $1^{\circ}$ and $30^{\circ}$ (below), applying FFT-based method. value and the corresponding measurement uncertainties at 95\% of confidence level. In particular, the SAM, introduces a measurement uncertainty contribution which corresponds to $0.8^{\circ}$, at $95 \%$ confidence level, and has been retrieved from 2.5 and 97.5 Monte Carlo distribution percentiles.

The analysis of the data showed that both the SAM and the $\mathrm{ADM}_{\text {SURF }}$ follow a quadratic trend, that is in good agreement with the results obtained through the analysis of the analytical model [32]. As reported in [31], if the difference $\left|\bar{M}_{1}-\bar{M}_{2}\right|$ has the same order of magnitude as, or even less than, the sum $\left(\delta_{T_{1}}+\delta_{T_{2}}\right)$, then measurements can be considered consistent, within the interval of the experimental uncertainties. From the data reported in Table 4, the differences between the mean values are less with respect to the sum of the correspondent total uncertainties, therefore the measurements can be considered compatible, confirming that the $\mathrm{ADM}_{\mathrm{SURF}}$ is suitable for the measurement of the angular displacement of the comb-drive of the MEMS microgripper under test. Moreover, it is important to underline that the computational complexity has been considerably reduced by using the $\mathrm{ADM}_{\mathrm{SURF}}$ : to process 390 images, as in our case, the SAM requires about 2 hours, instead the ADM SURF $_{\text {about 4-5 minutes only. }}$

As regards the data obtained with the $\mathrm{ADM}_{\mathrm{FFT}}$ (Figure 9), it emerged that the results do not follow a trend that can be closely related to the angular displacement of the comb-drive. From a first analysis, it can be deduced that, the $\mathrm{ADM}_{\mathrm{FFT}}$ cannot be considered suitable for the measurement of MEMS grippers whose angular displacement is below $1^{\circ}$, as there is no possibility of appreciating displacements around the tenth of a degree. Anyway, since some prototype of microgrippers, built with rotary comb-drives, are powered with voltages higher than $30 \mathrm{~V}$ and can be moved with angular displacements above $1^{\circ}$, the $\mathrm{ADM}_{\mathrm{FFT}}$ is evaluated also for an object that rigidly rotates around its axis by quantities greater than $1^{\circ}$. Table 3 , shows the measurement of rotation, $\mathrm{MoR}_{1}$, calculated applying $\mathrm{ADM}_{\mathrm{FFT}}$ to an image (see Figure 5), rotated of a quantity equal to $\mathrm{SET}_{1}$ and the measurement of rotation, $\mathrm{MoR}_{2}$, calculated applying the $\mathrm{ADM}_{\mathrm{FFT}}$ to the same image, rotated of a quantity equal to $\mathrm{SET}_{2}$, together with the angle measurement uncertainty $\delta_{\alpha}$, estimated from (3), considering $\alpha<15^{\circ}$ and from (5), considering $\alpha>15^{\circ}$. Test results confirm that angular displacements up to $30^{\circ}$ can be measured with an angle measurement uncertainty $\delta_{\alpha}$ lower than $0.02^{\circ}$, as can be seen in Table 3. The different behavior of the

Table 3. Measurement of rotation, $\mathrm{MoR}_{1}$, calculated applying ADMFFT to an image (see Figure 5), rotated of a quantity equal to $\mathrm{SET}_{1}$ and the measurement of rotation, $M_{2} R_{2}$, calculated applying ADMFFt to the same image, rotated of a quantity equal to $\mathrm{SET}_{2}$, together with the angle measurement uncertainty $\delta_{\alpha}$.

\begin{tabular}{cccccc}
\hline $\mathrm{SET}_{1}$ in $^{\circ}$ & MoR $_{\mathbf{1}}$ in $^{\circ}$ & $\delta_{\alpha}$ & $\mathrm{SET}_{\mathbf{2}}$ in $^{\circ}$ & $\mathrm{MoR}_{\mathbf{2}}$ in $^{\circ}$ & $\boldsymbol{\delta}_{\boldsymbol{\alpha}}$ \\
\hline 0.007 & 0 & 0.03 & 1 & 1.193 & 0.015 \\
0.032 & 0 & 0.015 & 2 & 2.767 & 0.015 \\
0.070 & 0 & 0.016 & 3 & 3.918 & 0.015 \\
0.120 & 0 & 0.015 & 4 & 4.029 & 0.015 \\
0.194 & 0 & 0.016 & 5 & 4.963 & 0.015 \\
0.277 & 0 & 0.015 & 7 & 5.078 & 0.015 \\
0.379 & 0 & 0.015 & 10 & 8.857 & 0.015 \\
0.497 & 0 & 0.015 & 13 & 8.127 & 0.016 \\
0.631 & 0.735 & 0.015 & 16 & 14.697 & 0.015 \\
0.777 & -0.262 & 0.015 & 20 & 16.571 & 0.015 \\
0.939 & 1.193 & 0.015 & 25 & 19.759 & 0.015 \\
1.118 & 1.193 & 0.015 & 30 & 29.237 & 0.015 \\
\hline
\end{tabular}


Table 4. Comb-drive angular displacement obtained through the SAM, SURF and FFT methods.

\begin{tabular}{|c|c|c|c|c|c|c|}
\hline Applied Voltage in V & SAM in ${ }^{\circ}$ & $\begin{array}{l}\text { Total Uncertainty } \\
\delta_{T_{S A M}} \text { in }^{\circ}\end{array}$ & SURF in ${ }^{\circ}$ & $\begin{array}{l}\text { Total Uncertainty } \\
\delta_{T_{S U R F}} \text { in }^{\circ}\end{array}$ & FFT in ${ }^{\circ}$ & $\begin{array}{l}\text { Total Uncertainty } \\
\delta_{T_{F F T}} \text { in }^{\circ}\end{array}$ \\
\hline 2 & 0.0 & 0.8 & 0.01 & 0.07 & 0.03 & 0.29 \\
\hline 4 & 0.0 & 0.8 & 0.04 & 0.08 & 0.21 & 0.23 \\
\hline 6 & 0.0 & 0.8 & 0.08 & 0.07 & 0.31 & 0.24 \\
\hline 8 & 0.1 & 0.8 & 0.14 & 0.07 & 0.22 & 0.28 \\
\hline 10 & 0.2 & 0.8 & 0.23 & 0.09 & 0.28 & 0.28 \\
\hline 12 & 0.3 & 0.8 & 0.33 & 0.08 & 0.23 & 0.31 \\
\hline 14 & 0.4 & 0.8 & 0.45 & 0.08 & 0.50 & 0.25 \\
\hline 16 & 0.5 & 0.8 & 0.56 & 0.19 & 0.68 & 0.23 \\
\hline 18 & 0.6 & 0.8 & 0.95 & 0.12 & 0.57 & 0.18 \\
\hline 20 & 0.8 & 0.8 & 0.89 & 0.10 & 0.10 & 0.21 \\
\hline 22 & 0.9 & 0.8 & 1.08 & 0.10 & 0.05 & 0.21 \\
\hline 24 & 1.1 & 0.8 & 1.27 & 0.16 & 0.04 & 0.18 \\
\hline
\end{tabular}

$\mathrm{ADM}_{\mathrm{FFT}}$ depending on the angular range can be deduced from results of the two rotation sets (Table 1) in Figure 10: for rotations below $1^{\circ}$, the least squares regression line has shown a $R^{2}=0.54$, while $R^{2}=0.97$ for angles between $1^{\circ}$ and $30^{\circ}$. In conclusion, it is possible to confirm that the $\mathrm{ADM}_{\mathrm{FFT}}$ is not suitable for the measurement of rotations below $1^{\circ}$, but for greater rotations (higher than $1^{\circ}$ ), it has an almost linear behavior.

\section{CONCLUSIONS}

This preliminary study has the purpose of comparing the measurements performed by different methods for the angular displacement of a comb-drive of a MEMS gripper prototype for biomedical applications. In particular, three in-house methods have been implemented in Matlab environment: the SAM, the $\mathrm{ADM}_{\text {SURF }}$ and the ADM $\mathrm{M}_{\mathrm{FFT}}$. Considering the SAM, the contribution of uncertainty related to the subjectivity of the operator has been estimated, which has found to be $0.8^{\circ}$, at $95 \%$ of confidence level, as previously indicated. From the experimental results obtained, it has been found that the SAM and $\mathrm{ADM}_{\mathrm{SuRF}}$ are suitable to measure the small angular displacement of the comb-drive of the microgripper, showing quadratic curves, consistent with the results obtained with the analytic model. Conversely, from the results retrieved by means of the $\mathrm{ADM}_{\mathrm{FFT}}$, it has been found no good correlation between small angular displacement and applied voltage that describe the real behavior of the device, and the data are not consistent with both the data obtained through the analytical method and with the two abovementioned methods.

Anyway, it was also assessed that this method was suitable for measuring rotations from $1^{\circ}$ to $30^{\circ}$, and a good correlation is observed between the $\mathrm{ADM}_{\mathrm{FFT}}$ outcomes and the rotations applied by the operator, with an uncertainty of about $0.02^{\circ}$. A comparison between the SAM and the $\mathrm{ADM}_{\mathrm{SURF}}$ has been proposed: the measurements can be considered compatible, confirming that the $\mathrm{ADM}_{\text {SURF }}$ is suitable for the measurement of the angular displacement of the comb-drive of the MEMS microgripper under test can be considered compatible. In particular the ADMSURF measurements of the comb-drive angular displacement are affected by an uncertainty lower than $8 \%$ for voltages less than $14 \mathrm{~V}$, as well as smaller than SAM. In conclusion, it can be confirmed that the $\mathrm{ADM}_{\mathrm{SURF}}$ is the most suitable method among the three proposed for the characterization of the angular displacement of MEMS devices such as microgrippers, both for the results obtained and the significant reduction of the computational costs.

\section{REFERENCES}

[1] Bhansali, Shekhar, Abhay Vasudev, eds. MEMS for biomedical applications. Elsevier, 2012, ISBN 978-0-85709-627-2.

[2] D. Panescu, Mems in medicine and biology, IEEE Eng. Med. Biol. Mag. 25 (2006), pp. 19-28.

DOI: $10.1109 / \mathrm{memb} .2006 .1705742$

[3] K. Keekyoung, X. Liu, Y. Zhang, Y. Sun, Nanonewton forcecontrolled manipulation of biological cells using a monolithic MEMS microgripper with two-axis force feedback, J. Micromech. Microeng. 18 (2008). DOI: $10.1088 / 0960-1317 / 18 / 5 / 055013$

[4] F. Vurchio, P. Ursi, A. Buzzin, A. Veroli, A. Scorza, M. Verotti, S. A. Sciuto, N. P. Belfiore, Grasping and Releasing Agarose micro Beads in Water Drops, Micromachines 10 (2019). DOI: $10.3390 / \mathrm{mi1} 0070436$

[5] A. Gosline, N. Vasilyev, E. Butler, C. Folk, A. Cohen, R. Chen, N. Lang, P. Del Nido, P. Dupont, Percutaneous intracardiac beatingheart surgery using metal mems tissue approximation tools, Int. J. Rob. Res. 31 (2012), pp. 1081-1093.

DOI: $10.1177 \% 2 \mathrm{~F} 0278364912443718$

[6] D. Benfield, S. Yue, E. Lou W. Moussa, Design and calibration of a six-axis mems sensor array for use in scoliosis correction surgery, J. Micromech. Microeng. 24 (2014). DOI: $10.1088 / 0960-1317 / 24 / 8 / 085008$

[7] F. Orsini, F. Vurchio, A. Scorza, R. Crescenzi, S. A. Sciuto, An Image Analysis Approach to Microgrippers Displacement Measurement and Testing, Actuators 7 (2018). DOI: $10.3390 /$ act 7040064

[8] F. Vurchio, P. Ursi, F. Orsini, A. Scorza, R. Crescenzi, S. A. Sciuto, N. P. Belfiore, Toward Operations in a Surgical Scenario: Characterization of a Microgripper via Light Microscopy Approach, Appl. Sci. 9 (2019). DOI: $\underline{10.3390 / \text { app9091901 }}$

[9] F. Vurchio, F. Orsini, A. Scorza, S. A. Sciuto, Functional characterization of MEMS Microgripper prototype for biomedical application: preliminary results, Proc. of 2019 IEEE International Symposium on Medical Measurements and Applications (MeMeA), Istanbul, Turkey, 26 - 28 June 2019. DOI: $10.1109 / \mathrm{MeMeA} .2019 .8802178$

[10] F. Vurchio, G. Fiori, A. Scorza, S. A. Sciuto, A comparison among three different image analysis methods for the displacement measurement in a novel MEMS device, Proc. of the 24th IMEKO TC4 International Symposium \& 22nd International Workshop on ADC Modelling and DAC Modelling and Testing, Palermo, Italy, 14 - 16 September 2020. Online [Accessed 18 January 2021]. https://www.imeko.org/publications/tc4-2020/IMEKO-TC42020-61.pdf 
[11] H. Bay, A. Ess, T. Tuytelaars, L. Van Gool, Speeded Up Robust Features (SURF), Computer Vision and Image Understanding, 110 (2008), pp. 346-359. DOI: $10.1016 /$ i.cviu.2007.09.014

[12] R. Bracewell, Fourier Analysis and Imaging, SpringerScience+Business Media, LLC, 2003.

[13] K. J. R. Liu, Pattern Recognition and Image Processing, Marcel Dekker, Inc.

[14] G. Dougherty, Digital Image Processing for Medical Applications, Cambridge University Press, 2009.

[15] R. C. Gonzalez, Digital Image Processing Using MATLAB, Pearson Prentice-Hall, 2004.

[16] W. Burger, M. J. Burge, Principles of Digital Image Processing, Springer.

[17] A. Bagolini, S. Ronchin, P. Bellutti, M. Chistè, M. Verrotti, N. P. Belfiore, Fabrication of Novel MEMS Microgrippers by Deep Reactive Ion Etching With Metal Hard Mask, Journal of Microelectromechanical Systems 26 (2017), pp. 926-934. DOI: $10.1109 /$ JMEMS.2017.2696033

[18] C. Potrich, L. Lunelli, A. Bagolini, P. Bellutti, C. Pederzolli, M. Verotti, N. P. Belfiore, Innovative Silicon Microgrippers for Biomedical Applications: Design, Mechanical Simulation and Evaluation of Protein Fouling, Actuators 7 (2018). DOI: $10.3390 /$ act7020012

[19] M. Verotti, A. Dochshanov, N. P. Belfiore, A Comprehensive Survey on Microgrippers Design: Mechanical Structure, J. Mech. Des. 139 (2017). DOI: $10.1115 / 1.4036351$

[20] R. Cecchi, M. Verotti, R. Capata, A. Dochshanov, G. B. Broggiato, R. Crescenzi, M. Balucani, S. Natali, G. Razzano, F. Lucchese, A. Bagolini, P. Bellutti, E. Sciubba, N. P. Belfiore, Development of Micro-Grippers for Tissue and Cell Manipulation with Direct Morphological Comparison, Micromachines 6 (2015), pp. 17101728.

DOI: $10.3390 / \mathrm{mi} 6111451$

[21] P. Di Giamberardino, A. Bagolini, P. Bellutti, I. J. Rudas, M. Verotti, F. Botta, N. P. Belfiore, New MEMS Tweezers for the Viscoelastic Characterization of Soft Materials at the Microscale, Micromachines 9 (2018). DOI: $10.3390 / \mathrm{mi} 9010015$

[22] M. Verotti, A. Dochshanov, N. P. Belfiore, Compliance Synthesis of CSFH MEMS-Based Microgrippers, J. Mech. Des. 139 (2017). DOI: $10.1115 / 1.4035053$

[23] F. Vurchio, F. Orsini, A. Scorza, F. Fuiano, S. A. Sciuto, A preliminary study on a novel automatic method for angular displacement measurements in microgripper for biomedical applications, Proc. of 2020 IEEE International Symposium on Medical Measurements and Applications (MeMeA), Bari, Italy, 1 June -1 July 2020. DOI: $10.1109 / \mathrm{MeMeA} 49120.2020 .9137249$

[24] M. Schaeferling, G. Kiefer, Object Recognition on a Chip: A Complete SURF-Based System on a Single FPGA, Proc. of 2011 International Conference on Reconfigurable Computing and FPGAs, Cancun, Mexico, 30 November - 2 December 2011. DOI: $10.1109 /$ ReConFig. 2011.65

[25] Q. Xu, Design, Fabrication, and Testing of an MEMS Microgripper With Dual-Axis Force Sensor, IEEE Sensors Journal, 15 (2015), pp. 6017-6026. DOI: 10.1109 /JSEN.2015.2453013

[26] M. Verotti, A. Bagolini, P. Bellutti, N. P. Belfiore, Design and Validation of a Single-SOI-Wafer 4-DOF Crawling Microgripper, Micromachines (Basel.) 10 (2019).

DOI: $10.3390 / \mathrm{mi1} 0060376$

[27] ISO/IEC Guide 98-3: 2008

[28] G. Fiori, F. Fuiano, A. Scorza, J. Galo, S. Conforto, S. A. Sciuto, Lowest Detectable Signal in medical PW Doppler Quality Control by means of a commercial Flow Phantom: a case study, Proc. of the 24th IMEKO TC4 International Symposium \& 22nd International Workshop on ADC Modelling and DAC Modelling and Testing, Palermo, Italy, 14 - 16 September 2020. Online [Accessed 14 June 2021]. https://www.imeko.org/publications/tc4-2020/IMEKO-TC4 2020-63.pdf

[29] G. Fiori, F. Fuiano, F. Vurchio, A. Scorza, M. Schmid, S. Conforto, S. A. Sciuto, A preliminary study on a novel method for Depth of Penetration measurement in Ultrasound Quality Assessment, Proc. of the 24th IMEKO TC4 International Symposium \& 22nd International Workshop on ADC Modelling and DAC Modelling and Testing, Palermo, Italy, 14 - 16 September 2020. Online [Accessed 14 June 2021]. https://www.imeko.org/publications/tc4-2020/IMEKO-TC42020-62.pdf

[30] C. H. Holbrow, J. N. Lloyd, J. C. Amato, E. Galvez, M. E. Parks, Modern Introductory Physics, Second Edition, Springer 2010.

[31] J. R. Taylor, An Introduction to Error Analysis. Uncertainty Stuty in Physical Measurements, Zanichelli: Bologna, Italy, 1986.

[32] R. Crescenzi, M. Balucani, N. P. Belfiore, Operational characterization of CSFH MEMS technology based hinges, J. Micromech. Microeng. 28 (2018). DOI: $\underline{10.1088 / 1361-6439 / \text { aaaf31 }}$ 